\title{
Building a Reference Transcriptome for the Hexaploid Hard Fescue Turfgrass (Festuca brevipila) Using a Combination of PacBio Isoseq and Illumina Sequencing
}

\section{CURRENT STATUS: UNDER REVIEW}

BMC Genomics BMC Series

Yinjie Qiu

University of Minnesota

ح qiuxx221@umn.eduCorresponding Author

ORCiD: https://orcid.org/0000-0002-0584-0688

Ya Yang

University of Minnesota

Cory D. Hirsch

University of Minnesota

Eric Watkins

University of Minnesota

DOI:

10.21203/rs.3.rs-18156/v1

SUBJECT AREAS

Plant Molecular Biology and Genetics

KEYWORDS

hard fescue, Pacbio Isoseq, RNA sequencing, phylogenetics, turfgrass 
Abstract

Background: Hard fescue (Festuca brevipila Tracey, $2 n=6 x=42$ ) is a cool season turfgrass with a fine leaf texture that performs well under low-input management. Breeding and genetics studies of $F$. brevipila have been limited due to the complexity of its hexaploid genome. To advance our knowledge of F. brevipila genomics, we used PacBio isoform sequencing to develop a reference transcriptome for this species.

Results: Here, we report the F. brevipila reference transcriptome generated from root, crown, leaf, and seed head tissues. We obtained 59,510 full-length transcripts, of which 38,595 were nonredundant full-length transcripts. The longest and shortest transcripts were 11,487 and $58 \mathrm{bp}$, respectively. To test the polyploid origin of $\mathrm{F}$. brevipila, we sequenced three additional transcriptomes using closely related species on an Illumina platform. The results of our phylotranscriptomic analysis supported the allopolyploid origin of F. brevipila.

Conclusions: Overall, the F. brevipila Pacbio Isoseq reference transcriptome provided the foundation for transcriptome studies and allowed breeders for gene discovery in this important turfgrass species. Full-text

Due to technical limitations, full-text HTML conversion of this manuscript could not be completed. However, the manuscript can be downloaded and accessed as a PDF. 